\title{
Dust Features in the Infrared Spectra of Planetary Nebulae
}

\author{
Kevin Volk \\ Dept. Physics and Astronomy, University of Calgary, Calgary, Alberta, \\ Canada T2N $1 N_{4}$
}

\begin{abstract}
I review some aspects of the infrared continuum emission of planetary nebulae, with particular emphasis on the various dust features. The original presentation with colour figures can be found at http://www.iras. ucalgary.ca/ volk/iau209.html.
\end{abstract}

\section{Introduction}

It is now more than 30 years since it was discovered that planetary nebulae $(\mathrm{PNe})$ often have large infrared excesses compared to what was expected from their optical and radio emission. This was first found for NGC 7027 (Gillett, Low \& Stein 1967) and subsequently was also found to be the case for 113 of the better known PNe (Cohen \& Barlow 1974). If one has only an ionized plasma producing the infrared emission, one would expect that the infrared spectra of PNe would be similar to their optical spectra, with a relatively weak boundfree/free-free continuum and strong emission lines. The general magnitude of the infrared continuum at any wavelength would be expected to be of order half the value of the $5 \mathrm{GHz}$ radio flux density: roughly 0.05 to $0.3 \mathrm{Jy}$ for most objects and of order $3 \mathrm{Jy}$ for the brightest objects. Instead what was found was much stronger infrared emission that generally peaks in the 25 to $60 \mu \mathrm{m}$ wavelength range. This was immediately attributed to emission from dust particles in and around the ionized region, with typical maximum temperatures of order $200 \mathrm{~K}$. While there are cases of $\mathrm{PNe}$ with little infrared excess, a large fraction of the known PNe have infrared excesses. We now have clear observational evidence that this dust is the remnant of the dust envelope of the star when it was on the asymptotic giant branch (AGB).

In the Infrared Astronomical Satellite (IRAS) mission in 1981 it was found that about $70 \%$ of the then-known PNe had detectable far-infrared emission, which in most cases had to be due to dust emission. Zhang and Kwok (1991) showed that in a sample of 66 of the better studied nebulae the average amount of infrared emission from the dust was $37 \% \pm 14 \%$ of the total bolometric luminosity. This sample is probably somewhat biased in favour of younger, and hence dustier, objects, yet it shows the general magnitude of the dust emission. This means that the dust optical depth at some wavelength, presumably in the ultraviolet, is of order 0.4 for an average nebula.

To illustrate the situation, Figure 1 shows the spectral energy distribution of NGC 7027 from radio to ultraviolet wavelengths, updated from Terzian (1989). I also show a nebular continuum fit based upon the radio data and 


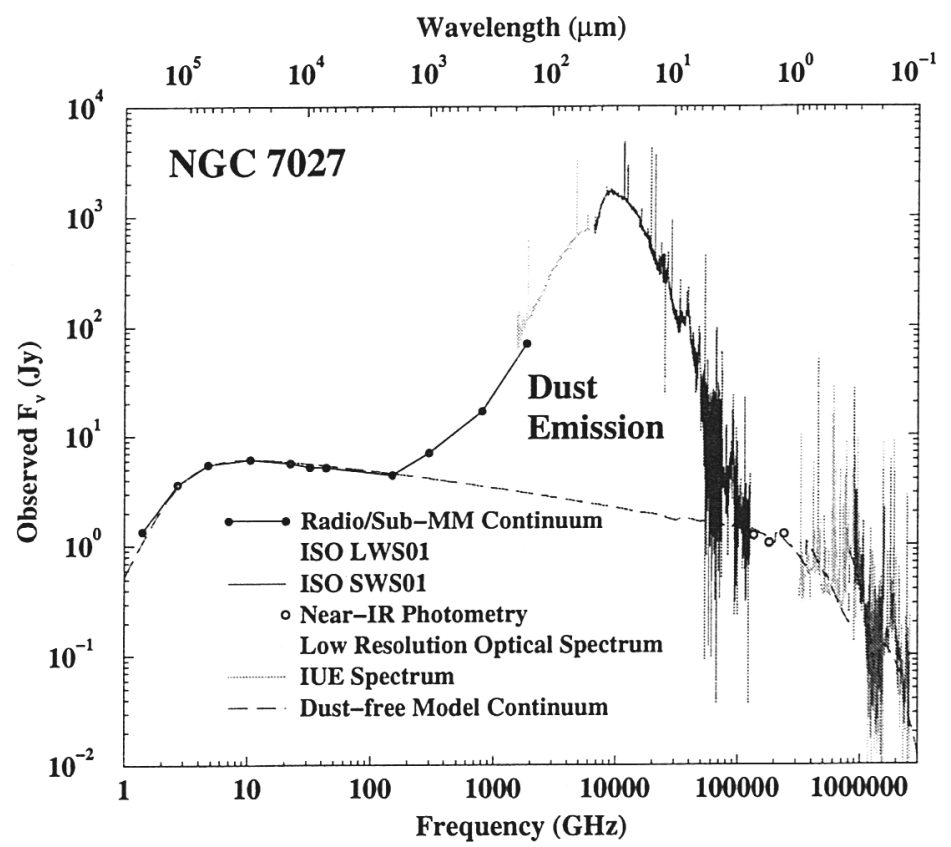

Figure 1. The spectral energy distribution of NGC 7027, after Terzian (1989). The dotted line shows a nebular continuum fit to the radio and optical data using a dust-free photoionization model, which fails badly in the infrared and sub-millimetre parts of the spectrum.

the optical/ultraviolet data, assuming a totally dust-free ionized region. While the model fits the radio and optical data rather well, it completely fails in the infrared and sub-millimetre wavelength range. One is simply unable to detect the extent of the dust emission from radio observations and optical/ultraviolet spectra. This is a problem because these are our primary diagnostic tools for studying PNe. One can gather some evidence of the effects of the dust by combining optical and radio images (for example see Lee, Kwok \& Lim, these proceedings) but this is not often done and in any case cannot be used for $\mathrm{PNe}$ which are too small to properly image.

Good reviews of the past work on the infrared emission from PNe can be found in Barlow (1992) and Roche (1989). Most of the issues these reviews discuss are still unresolved today.

\section{Issues Concerning the Dust in PNe}

We knows that there is dust associated with $\mathrm{PNe}$, but a number of questions have not yet been answered, including the following:

- Are the dust grains destroyed in or ejected from the ionized region?

- Where are the grains located in PNe?

- How much of an effect do the grains have on the physical properties of PNe? 
- How are the grains changed by exposure to the electron plasma and the ultraviolet radiation field of the central star?

Photoionization models show that the dust grains can have a significant effect on the physics of ionized regions (Baldwin et al. 1991, Dopita \& Sutherland 2000, Stazinska \& Szczerba 2002). A good observational example of this is the PN IRAS 18333-2357 in the globular cluster M22, where the dust is powering the nebula (Borkowski \& Harrington 1991). In more normal PNe it is not clear how much effect the dust actually has on the ionized region. Derived dust-togas mass ratios in PNe have tended to be somewhat smaller than what is found for the interstellar medium and for AGB stars, which implies at least partial dust destruction or ejection from the PN. However this is still being debated: see van Hoff et al. (2000) and Stazinska \& Szczerba (1999) for a discussion. It has been suggested that the dust grains can produce sufficient heating to cause temperature fluctuations across the ionized region, which are able to solve the $t^{2}$ problem in both PNe and HII regions. The more small dust grains one has the bigger the effect on the temperature distribution of the PN. If the dust grains are broken up by exposure to the electron plasma, this would tend to enhance the thermal effect of the dust.

The question of how the dust is distributed is more difficult to address observationally. It may be that all $\mathrm{PNe}$ have the equivalent of the cometary globules observed in NGC 7293, and that the dust and molecules are found in these condensations at a different density and temperature than that of the main ionized region. This would explain the rather puzzling observation that some $\mathrm{PNe}$ appear very similar in $\mathrm{H}_{2}$ line images and in the forbidden line images; if true this has important implications for the dust emission as well.

I will now discuss what I consider to be the two most important new areas of research on dust grains in PNe that have developed in the past few years: results from the Infrared Space Observatory (ISO) and high resolution groundbased imaging in the far-infrared using adaptive optics.

\section{Infrared Space Observatory Results}

Some preliminary ISO results concerning PNe were presented in the Groningen symposium. Since then the ISO calibration and data reduction have been much refined, and various results have appeared in the literature. A number of new dust features were discovered by ISO: see the review by Molster in these proceedings. There have also been some imaging results by ISOCAM (Persi et al. 1999). In this review I wish to look at the global properties of the dust emission using the spectroscopic data from ISO.

I have extracted the SWS and LWS data for $63 \mathrm{PNe}$ from the ISO archive, and examined these as to what features are present. This is a large fraction of all the good quality spectra of PNe from ISO, and they were taken for many different programs (in particular those of Mike Barlow and of Rens Waters). Results have been published for some objects (i.e. Salas et al. 2001; Molster et al. 2001) but not for many of these PNe.

Figure 2 shows the three general types of ISO spectra observed: some objects like A77 show no obvious dust features; some objects such as IC 418 show 
a variety of features associated with carbon-rich dust grains--the UIR ("PAH") features, the 21 and $30 \mu \mathrm{m}$ features, and the $11 \mu \mathrm{m}$ feature; and some objects such as M1-78 and CPD $-56^{\circ} 8032$ show features associated with oxygen-rich dust grains - crystalline and amorphous silicate features, water ice features, and so on. A few objects, such as NGC 2440 , show strongly line-dominated ISO spectra with little dust continuum which I will not discuss further. Out of the 63 objects I have looked at three have no detected dust emission, 16 have no clear dust features, 16 have only carbon-based dust features, 19 have only silicate features, and 9 objects have both UIR features and silicate features in their spectra. The last group are often considered to show both carbon-rich and oxygen-rich chemistries, which was not known prior to the ISO mission.
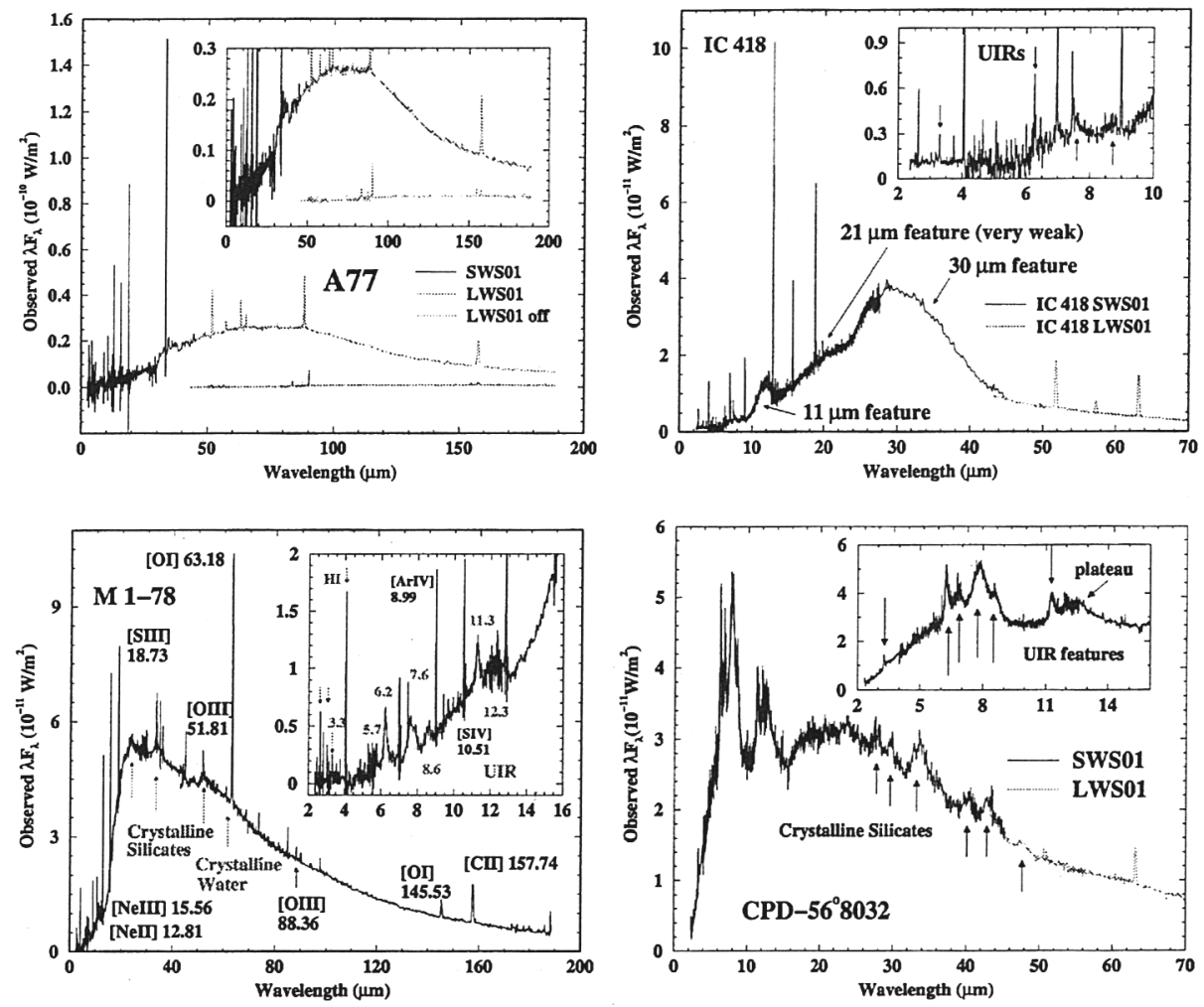

Figure 2. Examples of ISO spectra of PNe: A77 shows no obvious dust features although it has a significant dust continuum; IC 418 shows carbon-based features; M1-78 shows oxygen-based dust/ice features; CPD $-56^{\circ} 8032$ shows strong UIR features and crystalline silicates together.

One thing that I believe is quite significant is that one does not observe the silicate features together with the 11,21 , or $30 \mu \mathrm{m}$ features. If one simply has a nebula with some carbon-rich grains and some oxygen-rich grains, why is it that these carbon-based features are never seen with the crystalline silicates? Since 
the UIR features are attributed to some type of large carbon-ring molecules, similar to PAHs, what we see is that these molecules but not the bulk grains that produce the other carbon-based dust features can co-exist with crystalline silicates. Presonally I think that the UIRs are sometimes formed in an oxygenrich environment, as may be true for the Orion Nebula, and cannot be taken to definitively mark a carbon-rich chemistry - especially when the UIR features are weak. However for some objects such as CPD $-56^{\circ} 8032$ with strong UIR emission and crystalline silicates one likely does have a mixed chemistry.

Another noteworthy result of this sample is that the $11 \mu \mathrm{m}$ feature which is attributed to $\mathrm{SiC}$ grains is relatively rare, in contrast to the case for carbon stars. Only two of the PNe, IC 418 and NGC 6790, have strong $11 \mu \mathrm{m}$ emission features. It appears that this feature is also changed compared to what is seen in carbon star spectra-the peak wavelength is longer and the feature width is larger than what is normally seen in carbon star spectra. Thus here we have what appears to be an example of feature evolution after the AGB phase. Whether this is due to a change in the grain size or the grain composition is worthy of further study.

If one looks at all objects showing some carbon-based spectral feature, then first it is seen that almost all of them (23 of 24 sources, the other object being NGC 6790) have the UIR features. Nine of these 23 sources have crystalline silicates while ten have the $30 \mu \mathrm{m}$ feature. The remaining four show only the UIRs, although some of these sources simply have poorer ISO data and so may have other features as well. I see the $21 \mu \mathrm{m}$ feature only in the spectrum of IC 418 , which has an unusually strong $30 \mu \mathrm{m}$ feature for PNe. Hony, Waters \& Tielens (2001) state that NGC 40 and NGC 6369 have the $21 \mu \mathrm{m}$ feature. I am not entirely certain of the reality of the feature in these objects, but in any case it must be rather weak if present.

In the silicate group, the most noteworthy property is that a large majority of the sources (24 of 28 ) have crystalline silicate features and few have only amorphous silicate emission features. If one assumes that a circumbinary disk is needed to produce the crystalline silicates, then the large fraction of objects with these features is important in the question of how frequently $\mathrm{PNe}$ are the product of binary evolution. Nine of the objects have the UIR features, which is about $1 / 3$ of the group.

The objects with no clear dust features seems likely to include a number of sources with silicate dust grains where there is little crystalline silicate and where the dust temperature is too low for the $10 \mu \mathrm{m}$ amorphous silicate feature to be obvious. Some of these sources may have weak $33.7 \mu \mathrm{m}$ crystalline silicate features, but with a strong $33.5 \mu \mathrm{m}$ [SIII] line on top of the possible features it is difficult to be certain that they are real. In some of these sources the dust continuum is simply rather weak; yet in other cases, including A77, the dust continuum is quite strong but one still does not see any obvious features. Some of the latter objects could have carbon-rich dust but lack the 11,21 , and $30 \mu \mathrm{m}$ features. If so, it would be interesting to find out why the features are missing since that should help to identify the carrier grains.

One can attempt to make a dust emission model to fit the ISO data for different objects (see Pei and Volk, these proceedings) but this is unlikely to give a unique result without having infrared images as well as spectral information 
to fit. Since the electron plasma contributes a local heating source for the dust grains, which is heavily dependent on the (unknown) dust grain surface properties, any dust emission model is more difficult than for AGB stars where one knows that the star is the only heating source. I think that combining ISO spectra and ground-based infrared images of PNe will give us the most insights into the nature of the dust grains in the next few years.

\section{Ground Based Imaging}

There has been a long history of ground-based infrared imaging of PNe, in particular to attempt to trace the source regions of the UIR features (see for example Woodward et al. 1989; Hora et al. 1993; Ueta et al. 2001) at 3.3, 7.6, and $11.3 \mu \mathrm{m}$. What is new at the present time is the use of adaptive optics on 8-metre class telescopes optimized for infrared observations. This combination provides significantly better angular resolution and sensitivity than was previously possible, and extends the wavelength range for observations out to about $25 \mu \mathrm{m}$. With narrow-band filters one has the potential to isolate various features: the $11 \mu \mathrm{m} \mathrm{SiC}$ feature, the $10 \mu \mathrm{m}$ silicate feature, and potentially one or more of the crystalline silicate features in the Q-band window.

Recently Sun Kwok and I used the Gemini North telescope and the OSCIR infrared camera to image NGC 7027 and $\mathrm{BD}+30^{\circ} 3639$ in various narrow-band filters in the N-band and Q-band windows. The images can be found at the WWW address cited in the abstract. With three narrow-band filters at 10.3, 11.7 , and $12.5 \mu \mathrm{m}$ it is possible to map the nebulae in the $11.3 \mu \mathrm{m}$ UIR feature. Figure 3 shows the original three images and the continuum-subtracted $11.3 \mu \mathrm{m}$ image for NGC 7027. As was previously known, the UIR emission occurs just outside the ionized region for both objects, so the UIR carrier is destroyed in the ionized region; what is new is that we have high angular resolution $\left(0.4^{\prime \prime}\right)$ maps of the emission regions which we can compare with images at other wavelengths.

The possibility of carrying out this type of observation for the other features mentioned above is very exciting since combining a map of the emission region with the ISO spectral information to do a detailed photoionization and dust model of a PN gives us the chance to get additional information beyond what spectral fitting alone can provide: in particular if one knows the exact position where the features arise one can constrain the dust grain size distribution. This is possible because for a given radiation field smaller grains will be hotter than larger ones. Without spatial information one can fit the same spectrum with either larger grains closer in or smaller grains further out in the nebula. One would also like to correlate the dust emission regions with the nebular electron temperature to see whether in regions with hotter grains these are contributing to the heating of the plasma. For objects of apparent mixed chemistries, one wants to see if the carbon-based and oxygen-based grains are spatially separated.

\section{Future Prospects}

For the next few years I believe that infrared imaging will give us the most new information about dust in PNe. In particular if we can get an image of the crystalline silicate source region in an object such as CPD $-56^{\circ} 8032$ or NGC 

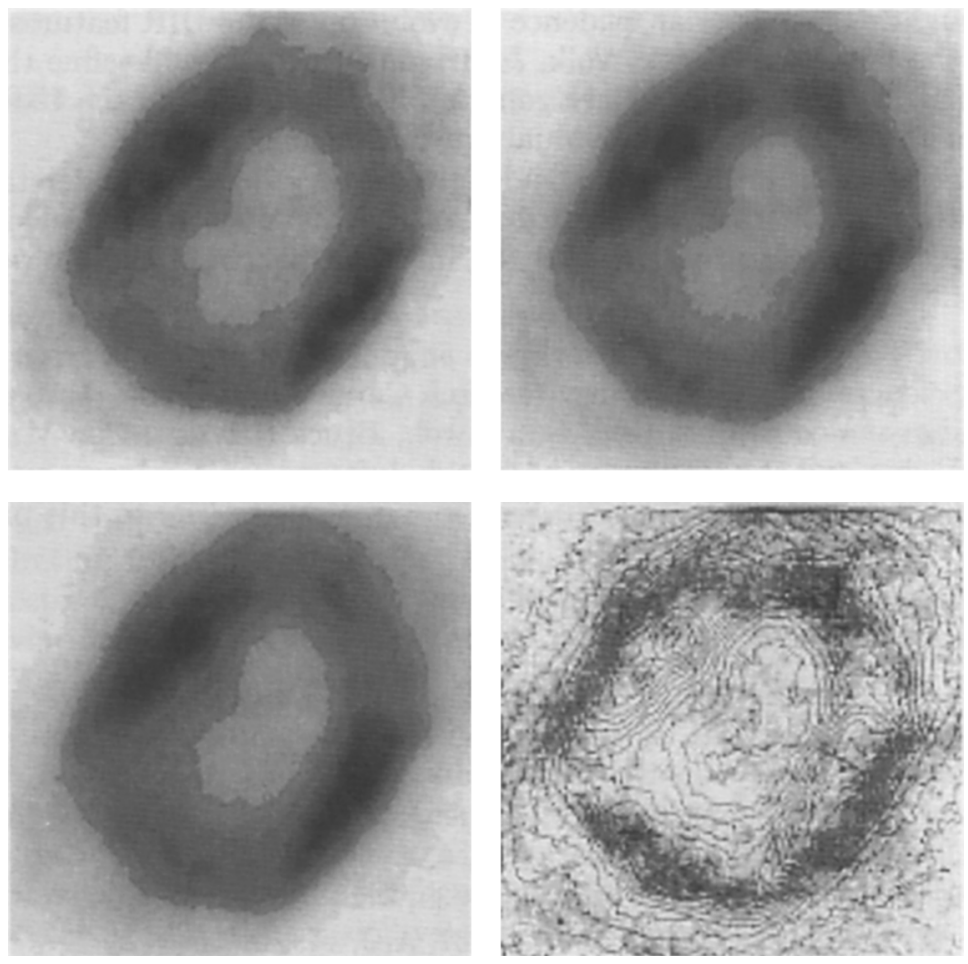

Figure 3. Gemini observations of NGC 7027 using the OSCIR camera: the upper left, upper right, and lower left panels show the 10.3, 11.7 , and $12.5 \mu \mathrm{m}$ filter images and the lower right panel shows the continuum-subtracted $11.7 \mu \mathrm{m}$ image with the continuum contours overlayed. The images are all $11^{\prime \prime}$ square; north is up and east is at left. The greyscales show sky brightness in Jy/square arc-second.

6302 one will immediately be able to tell whether the silicates are in some type of circumbinary disk or are from the equivalent of an Oort cloud around the PN. It would also be rewarding to try to trace the source region of the $11 \mu \mathrm{m}$ feature in IC 418 and NGC 6790 to see whether this sheds light on why the feature has changed since the AGB.

There is also the prospect of additional spectral observations of the dust features either from an airbourne observatory (i.e. SOFIA) or from space (i.e. the proposed AstroBiology Explorer). It is thought that the peak wavelength of the crystalline silicate features shifts with temperature, so one may get intrinsic dust temperature information directly from the features. Such information would be useful to understand the relation between all the new mineral species that ISO has discovered in PNe.

Finally, there is still a place for high spectral resolution ground-based observations of dust features. ISO failed to show any structure in the UIRs and other infrared dust features. At higher resolution we should be able to find structure in the UIR features if these are due to PAH-like molecules as has long 
been thought. There is clear evidence of evolution of the UIR features from the AGB to the PN stage (Kwok, Volk, \& Hrivnak 1999). It will refine the picture of the chemical processes that are going on if one can resolve the UIR features to look at the vibrations of individual types of chemical bonds.

All of these lines of study should give a very detailed understanding of exactly what is going on with the dust in $\mathrm{PNe}$ as they evolve, which will be a big challenge for future models of the infrared spectra of these objects both individually and collectively.

Acknowledgments. This work was supported in part by a grant from the Natural Sciences and Engineering Research Council of Canada. I would like to acknowledge the contributions of Sun Kwok, Bruce Hrivnak, Ben Warrington, R. Scott Fisher and the University of Florida infrared camera team, and the ISO SWS and LWS science teams (who are too numerous to list) to this paper.

\section{References}

Baldwin, J. A., et al. 1991, ApJ, 374, 580

Barlow, M. J. 1992, in I.A.U. Symposium 180 Planetary Nebulae ed.

R. Weinberger \& A. Acker (Kluwer:Dordrecht), 163

Borkwoski, K. J., \& Harrington, J. P. 1991, ApJ, 379, 168

Cohen, M., \& Barlow, M. J. 1974, ApJ, 193, 401

Dopita, M., \& Sutherland, R. S. 2000, ApJ, 539, 742

Gillett, F. C., Low, F. J., \& Stein, W. A. 1967, ApJ, 149, L97

Hony, S., Waters, L. B. F. M., \& Tielens, A. G. G. M. 2001, A\&A, 378, 41

Hora, J. L., et al. 1993, ApJ, 413, 304

Kwok, S., Volk, K., \& Hrivnak, B. J. 1999, A\&A, 350, L35

Molster, F., et al. 2001, A\&A, 372, 165

Roche, P. 1989, in I.A.U. Symposium 131 Planetary Nebulae ed. S. TorresPeimbert (Kluwer:Dordrecht), 117

Persi, P., et al. 1999, A\&A, 351, 201

Salas, J. B., et al. 2001, A\&A, 367, 949

Stazinska, G., \& Szczerba, R. 1999, A\&A, 351, 297

Stazinska, G., \& Szczerba, R. 2002, A\&A, in press

Terzian, Y. 1989, in I.A.U. Symposium 131 Planetary Nebulae ed. S. TorresPeimbert (Kluwer:Dordrecht), 17

Ueta, T., et al. 2001, in Post AGB Objects as a Phase of Stellar Evolution ed. R. Szczerba \& S. Gorny, (PASP:San Francisco), 339

van Hoff, P., et al. 2000, ApJ, 532, 384

Woodward, C. E., et al. 1989, ApJ, 342, 860

Zhang, C.-Y., \& Kwok, S. 1991, A\&A, 250, 179 